\title{
Comparison of the E test and a proportion dilution method for susceptibility testing of Mycobacterium avium complex
}

\author{
W. FABRY, E. N. SCHMID and R. ANSORG \\ Institute of Medical Microbiology, University of Essen, Hufelandstrasse 55, D-45147 Essen, Germany
}

\begin{abstract}
The newly developed $\mathbf{E}$ test was compared with an extended $1 \%$ proportion dilution method for determining the susceptibility of Mycobacterium avium complex (MAC) strains to amikacin, streptomycin, fusidic acid, rifampicin, clarithromycin, ciprofloxacin, ofloxacin and fleroxacin. For all antibiotics tested except clarithromycin and ciprofloxacin, no more than one strain gave a different susceptibility result with the two methods. The discrepant results occurred near the chosen breakpoint concentration of clarithromycin and outside the concentration range of the $E$ test for ciprofloxacin. For the minimum inhibitory concentration (MIC) values obtained within the range of antibiotic concentrations tested, there was good correlation between the two methods; the MICs differed by more than one two-fold dilution in no more than two strains per antibiotic. It is concluded that the $E$ test is suitable for susceptibility testing of MAC.
\end{abstract}

\section{Introduction}

Currently, strains of the M. avium complex (MAC) are the most commonly encountered mycobacteria isolated in most clinical laboratories [1]. This correlates with the increasing number of AIDS patients [2]. In contrast to $M$. tuberculosis susceptibility testing, results for MAC based on breakpoint testing methods, including the proportion method and the radiometric method with the BACTEC instrument, do not correlate well with clinical outcome [1]. Therefore, a simple method for the determination of minimum inhibitory concentrations (MICs) is required to investigate the relationship between in-vitro susceptibility testing and clinical outcome. The recently developed $\mathrm{E}$ test is a diffusion method of determining MICs which uses a plastic strip containing a pre-formed logarithmic gradient concentration of antimicrobial agent. For mycobacteria it has been evaluated only for the rapidly growing species $M$. fortuitum and $M$. chelonae [3]. However, a recent study has suggested that it is also suitable for testing the slowly growing species $M$. kansasii [4], and sharp inhibition zones have been shown to be produced after incubation for 2-3 weeks. The aim of this study was to investigate the suitability of $E$ tests, in comparison with

Received 20 March 1995; revised version accepted 9 Aug. 1995. an extended $1 \%$ proportion dilution method [5], for the determination of MICs of a range of agents for MAC.

\section{Materials and methods}

\section{Strains}

Twenty isolates of MAC cultured from different patients during 1993 were tested. They were identified by standard bacteriological culture methods [1] and confirmed by the AccuProbe ${ }^{\mathrm{TM}}$ nucleic acid hybridisation system (DPC Biermann GmbH, Germany). Reference strain ATCC 43216 was used for internal quality control.

\section{Antibiotics and breakpoint concentrations}

The following antibiotics were tested: amikacin (Bristol-Myers Squibb, Germany), streptomycin (Heyl, Germany), fusidic acid (Thomae, Germany), rifampicin (Grünenthal, Germany), clarithromycin (Abbott), ciprofloxacin (Bayer, Germany), ofloxacin (Hoechst, Germany) and fleroxacin (Hoffmann LaRoche, Switzerland). Antibiotic powders of defined activity were provided by the manufacturers of each agent. E test strips of fleroxacin were provided by Hoffmann LaRoche and ofloxacin strips by Hoechst. The other strips were purchased from Difco. The values for maximum achievable concentrations of the agents in 
serum vary in the literature [5-14]. Based on these, the following MICs $(\mathrm{mg} / \mathrm{L})$ were chosen as breakpoints for susceptibility for both methods: amikacin 8 , streptomycin 8 , fusidic acid 64 , rifampicin 32 , clarithromycin 8 , ciprofloxacin 8 , ofloxacin 8 and fleroxacin 8 .

\section{Proportion dilution method}

Amikacin, streptomycin, fusidic acid, rifampicin, clarithromycin, ciprofloxacin, ofloxacin and fleroxacin were dissolved in $\mathrm{NaCl} 0.85 \%$ solution. Rifampicin and clarithromycin were dissolved in small amounts of $\mathrm{N}, \mathrm{N}$-dimethylformamide (Merck, Germany) before the $\mathrm{NaCl}$ solution was added. The antibiotic solutions were added to Löwenstein-Jensen (L-J) medium. The standard proportion method for mycobacteria, with a single breakpoint concentration [5], was extended to four concentrations to achieve a better comparison with the $\mathrm{E}$ test. The final concentrations $(\mathrm{mg} / \mathrm{L})$ were: 0.5 , 2,8 and 32 for streptomycin, clarithromycin, ciprofloxacin, olfoxacin and fleroxacin; 2, 8, 32 and 64 for amikacin and rifampicin; and 8, 32, 64 and 128 for fusidic acid. The L-J medium containing antibiotics (3.5 ml of mixture/tube) was coagulated in a slant position by heating at $85^{\circ} \mathrm{C}$ for $30-40 \mathrm{~min}$. Tubes with L-J medium without antibiotics were used as growth controls.

The inoculum was prepared within a biological safety cabinet. Strains were cultured in liquid medium without Tween [15] for 1-2 weeks to achieve logarithmic growth, and this was then adjusted to give an OD560 of $0.15-0.2$. The antibiotic-containing and control media were inoculated with $c .0 .1 \mathrm{ml}$ of a $10^{2}$ diluted suspension, corresponding to $\left(1 \times 10^{4}\right)-$ $\left(2 \times 10^{4}\right) \mathrm{cfu}$. Further control tubes were inoculated with $10^{3}$ and $10^{4}$ dilutions of the suspension to achieve control tubes with an easily countable number of colonies [5]. The tubes were incubated for 3 weeks in the dark at $37^{\circ} \mathrm{C}$, the first week horizontally and the following 2 weeks vertically. They were examined for bacterial growth after incubation for 2 and 3 weeks.

The lowest concentration showing no bacterial growth, or $<1 \%$ of the number of colonies present in the $10^{4}$ dilution control tube, was recorded as the MIC.

\section{E test}

Petri dishes, $9 \mathrm{~cm}$ in diameter, were filled with $30 \mathrm{ml}$ of L-J medium. After coagulation of the medium they were pre-incubated at $37^{\circ} \mathrm{C}$ for 1 day to dry. The same inoculum was used for both the $E$ test and the proportion method. Approximately $0.2 \mathrm{ml}$ of the $10^{2}$ diluted suspension was applied with a pipette and spread over the surface of the medium with a sterile cotton swab. One E strip was placed on to each plate. The plates were sealed in plastic bags and incubated in the dark for 3 weeks at $37^{\circ} \mathrm{C}$. The MIC was recorded as the value on the $\mathrm{E}$ strip at the intersection with the zone of inhibition of bacterial growth. If there was a difference between the place of intersection on the right and left side of the strip, the higher concentration was recorded as the MIC. The MIC was determined according to the instructions of the manufacturer, i.e., where growth was completely inhibited: isolated colonies within the ellipse, which were very rarely seen, were ignored.

\section{Results}

The $\mathrm{E}$ test produced sharp inhibition zones after incubation for 3 weeks enabling the MICs to be read easily (Fig. 1). Some strains gave a result after incubation for 2 weeks with no change in the reading after 3 weeks. Double inhibition zones occurred in some cases with quinolones. In these cases the smaller inhibition zone was recorded. The MIC values obtained with the $\mathrm{E}$ test and the extended proportion dilution method are given in Table 1. The majority of MICs were outside the concentration range tested in both methods, but where the MIC was within the tested range no more than two strains for each antibiotic gave discrepancies of more than one dilution step between the two methods. The qualitative susceptibility results from both tests (i.e. resistants versus susceptible), based on the chosen breakpoints, are given in Table 2 . For clarithromycin, three discrepant results occurred near the breakpoint concentration. Four discrepant results were detected for ciprofloxacin, three of these isolates had MICs outside the concentration range of the $\mathrm{E}$ test. For the other antibiotics, no more than one strain per antibiotic gave different susceptibility results with the two methods (Table 2).

\section{Discussion}

The E test was evaluated for its ability to determine MICs of a range of antibiotics for MAC isolates and the results were compared with those from an extended proportion method. The E test strips produced sharp inhibition zones after incubation for 2-3 weeks. This reflects a more stable concentration gradient than the common paper disk method. A concentration gradient induced by paper disks is only suitable for testing rapidly growing bacteria. Slowly growing MAC isolates need a more stable gradient for sharp inhibition zones, as provided by the $\mathrm{E}$ test. Therefore, the $\mathrm{E}$ test is suitable for the determination of MICs for MAC. A large number of tubes would be necessary for MIC determinations by the proportion method, which is usually performed at a single breakpoint concentration. MIC determination with the BACTEC instrument would similarly require a large number of bottles [16]. In addition, if liquid medium 12B is used with the BACTEC, MAC strains appear generally susceptible to the primary anti-tuberculosis drugs, with the exception of isoniazid. This is in contrast to conventional 


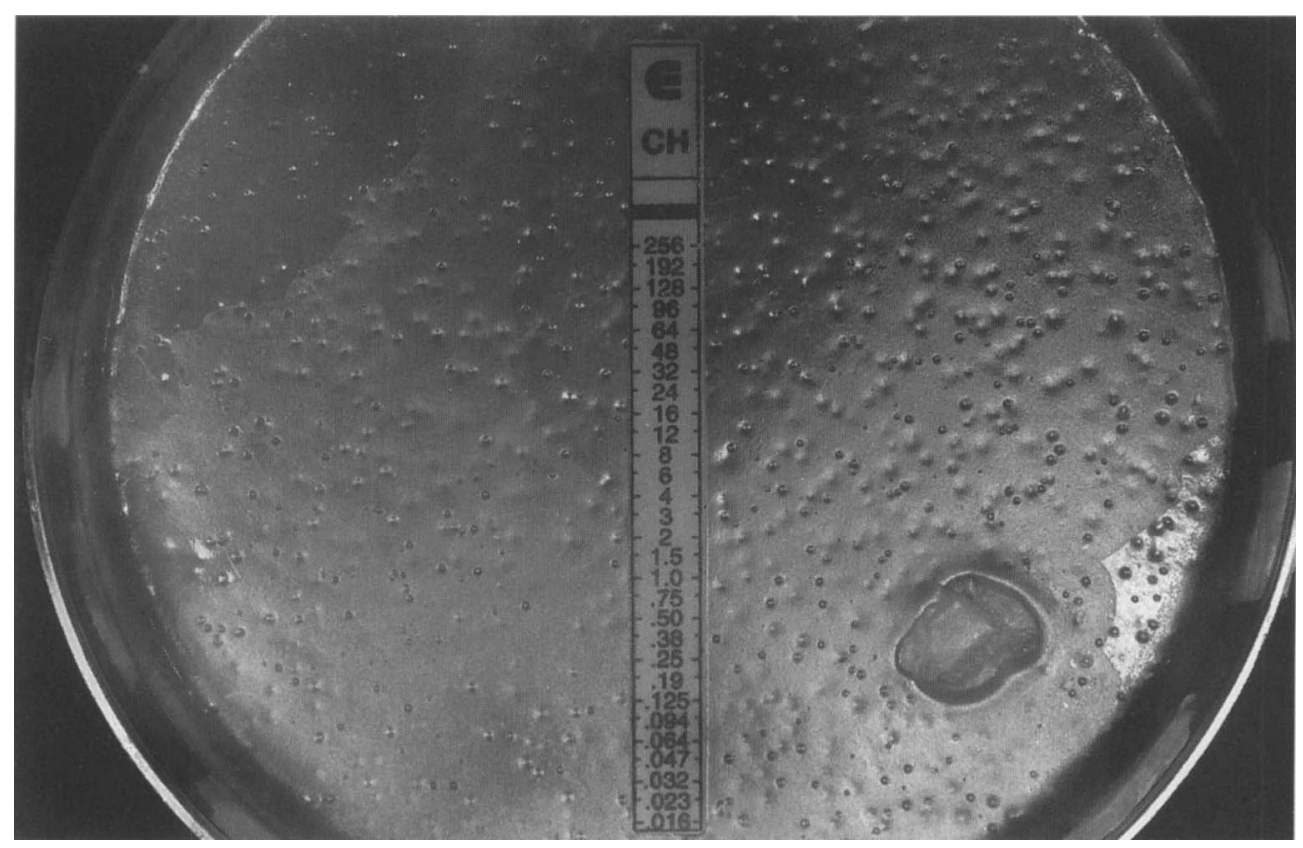

Fig. 1. $M$. avium complex grown for 3 weeks at $37^{\circ} \mathrm{C}$ on Löwenstein-Jensen medium. Zone of inhibition by clarithromycin with $\mathrm{E}$ test strip (MIC $6 \mathrm{mg} / \mathrm{L}$ ).

Table 1. MIC of several antibiotics for $20 \mathrm{MAC}$ isolates determined by the $\mathrm{E}$ test and proportion methods

\begin{tabular}{|c|c|c|c|c|c|c|c|c|}
\hline \multirow[b]{2}{*}{ Method } & \multicolumn{8}{|c|}{ Antibiotic $\mathrm{MIC}(\mathrm{mg} / \mathrm{L})$} \\
\hline & $\mathrm{AK}$ & SM & RI & FU & $\mathrm{CH}$ & $\mathrm{CI}$ & OF & FO \\
\hline \multicolumn{9}{|l|}{ E-test } \\
\hline $\mathrm{MIC}_{50}$ & 128 & 32 & $>256$ & $>256$ & 8 & $>32$ & $>32$ & $>256$ \\
\hline $\mathrm{MIC}_{90}$ & $>256$ & $>256$ & $>256$ & $>256$ & $>256$ & $>32$ & $>32$ & $>256$ \\
\hline Range & $8 \rightarrow>256$ & $6->256$ & $2->256$ & $96->256$ & $0.5->256$ & $32->32$ & $>32$ & $24->256$ \\
\hline \multicolumn{9}{|c|}{ Proportion method } \\
\hline $\mathrm{MIC}_{50}$ & 64 & 32 & $>64$ & $>256$ & 8 & 32 & $>32$ & $>32$ \\
\hline $\mathrm{MIC}_{90}$ & $>64$ & $>32$ & $>64$ & $>256$ & $>32$ & 32 & $>32$ & $>32$ \\
\hline Range & $8->64$ & $8->32$ & $\leqslant 2->64$ & $128->256$ & $\leqslant 0.5->32$ & $8-32$ & $8->32$ & $8->32$ \\
\hline
\end{tabular}

AK, amikacin; SM, streptomycin; RI, rifampicin; FU, fusidic acid; CH, clarithromycin; CI, ciprofloxacin; OF, ofloxacin; FO, fleroxacin; $\mathrm{MIC}_{50}$, minimum inhibitory concentration for $50 \%$ of the strains; $\mathrm{MIC}_{90}$, minimum inhibitory concentration for $90 \%$ of the strains.

Table 2. Susceptibility of MAC isolates by proportion dilution and E test

\begin{tabular}{lcccccccc}
\hline & \multicolumn{7}{c}{ Number of isolates } \\
\cline { 2 - 8 } Susceptibility & AK & SM & RI & FU & CH & CI & OF & FO \\
\hline $\begin{array}{l}\text { Susceptible by } \\
\text { both methods }\end{array}$ & 1 & 1 & 2 & 0 & 11 & 0 & 0 & 0 \\
$\begin{array}{l}\text { Resistant by } \\
\text { both methods }\end{array}$ & 18 & 19 & 18 & 20 & 6 & 16 & 19 & 19 \\
\begin{tabular}{l} 
Discrepant \\
\hline
\end{tabular} & $1^{\text {D }}$ & 0 & 0 & 0 & $3^{\text {S }}$ & $4^{\mathrm{S}}$ & $1^{\mathrm{s}}$ & $1^{\mathrm{S}}$ \\
\hline
\end{tabular}

Antibiotic abbreviations as for Table 1; D, resistant by proportion dilution method; $\mathrm{S}$, resistant by $\mathrm{E}$ test..

proportional dilution testing, which indicates more resistance [1]. However, the clinical relevance of invitro susceptibility results from either method is uncertain, particularly for results in the highly resistant category based on conventional tests [17]. This underlines the necessity for further MIC determinations in this range, which could ultimately lead to the evaluation of new breakpoints, perhaps separately for solid and liquid media. For comparison purposes the same breakpoint concentrations, chosen to reflect maximum achievable serum levels, were selected for both methods in this study and identical breakpoint 
concentrations were preferred for all quinolones [14]. In general, there was good agreement between the two methods in the resistant/susceptible evaluation. Where MIC values were distributed over the whole range of antibiotic concentrations tested, there was also good correlation between the results.

Conventional methods for the isolation and identification of MAC with solid media require at least 3 weeks and susceptibility testing of isolates may take another 3 weeks before results are available. Therefore, efforts should be made to reduce this time. The $\mathrm{E}$ test is a simple, convenient method suitable for MIC determination of agents for MAC and could be a useful tool for direct testing of microscopically positive clinical specimens. It is concluded that further current or putative anti-MAC agents could be tested by the $\mathrm{E}$ test, when strips are available.

\section{References}

1. Hawkins JE, Wallace RJ, Brown BA. Antibacterial susceptibility tests: mycobacteria. In: Balows A, Hausler W, Herrmann $\mathrm{K}$, Isenberg $\mathrm{H}$, Shadomy $\mathrm{H}$ (eds) Manual of clinical microbiology, $5^{\text {th }}$ edn. American Society for Microbiology, Washington. 1991: 304-339, 1138-1152.

2. Inderlied CB, Kemper CA, Bermudez, LEM. The Mycobacterium avium complex. Clin Microbiol Rev 1993; 6: 266-310.

3. Hoffner SE, Klintz L, Olsson-Liljequist B, Bolmström A. Evaluation of $\mathrm{E}$ test for rapid susceptibility testing of Mycobacterium chelonae and M. fortuitum. J Clin Microbiol 1994; 32: 1846-1849.

4. Fabry W, Schmid EN, Ansorg R. Comparison of the E test and a proportion dilution method for susceptibility testing of Mycobacterium kansasii. Chemotherapy 1995; 41: 247-252.

5. DIN 58943, Deutsches Institut für Normierung e. V. Medical microbiology; diagnosis of tuberculosis; methods for the determination of susceptibility of tubercle bacilli to chemotherapeutic agents 2nd edn. Berlin, Köln, Beuth Verlag. 1991: 264.

6. Inderlied CB. Antimycobacterial agents: in vitro susceptibility testing, spectrums of activity, mechanisms of action and resistance, and assays for activity in biological fluids. In: Lorian V (ed) Antibiotics in laboratory medicine, 3rd edn. Baltimore, Williams and Wilkins Co. 1991: 134-197.

7. Fenlon $\mathrm{CH}$, Cynamon $\mathrm{MH}$. Comparative in vitro activities of ciprofloxacin and other 4-quinolones against Mycobacterium tuberculosis and Mycobacterium intracellulare. Antimicrob Agents Chemother 1986; 29: 386-388.

8. Heifets L, Lindholm-Levy P. Comparison of bactericidal activities of streptomycin, amikacin, kanamycin, and capreomycin against Mycobacterium avium and $M$. tuberculosis. Antimicrob Agents Chemother 1989; 33: 1298-1301.

9. Leysen DC, Haemers A, Pattyn SR. Mycobacteria and the new quinolones. Antimicrob Agents Chemother 1989; 33: 1-5.

10. Rastogi N, Goh KS. Effect of $\mathrm{pH}$ on radiometric MICs of clarithromycin against 18 species of Mycobacteria. Antimicrob Agents Chemother 1992; 36: 2841-2842.

11. Reeves DS. The pharmacokinetics of fusidic acid. $J$ Antimicrob Chemother 1987; 20: 467-476.

12. Stone MS, Wallace RJ, Swenson JM, Thornsberry C, Christensen LA. Agar disk elution method for susceptibility testing of Mycobacterium marinum and Mycobacterium fortuitum complex to sulfonamides and antibiotics. Antimicrob Agents Chemother 1983; 24: 486-493.

13. Wise R, Lister D, McNulty CAM, Griggs D, Andrews JM. The comparative pharmacokinetics of five quinolones. $J$ Antimicrob Chemother 1986; 18 Suppl D: 71-81.

14. Klopman G, Wang S, Jacobs MR, Bajaksouzian S, Edmonds $\mathrm{K}$, Ellner JJ. Anti-Mycobacterium avium activity of quinolones: in vitro activities. Antimicrob Agents Chemother 1993; 37: 1799-1806.

15. Meißner G, Schmiedel A. Mykobakterien und mykobakterielle Erkrankungen. Jena, Gustav Fischer Verlag. 1968: 59.

16. Lee C-N, Heifets LB. Determination of minimum inhibitory concentrations of antituberculosis drugs by radiometric and conventional methods. Am Rev Respir Dis 1987; 136: 349-352.

17. Bailey WC, Bass JB, Hawkins JE, Kubica GP, Wallace RJ. Drug susceptibility testing for mycobacteria. Am Thorac Soc News 1984; 10: 9-10. 